\title{
A Relevância da Dosagem do Hormônio Estimulante da Tireóide em Pacientes com Fibromialgia
}

\section{The Relevance of Thyroid Stimulating Hormone Measure in Patients with Fibromyalgia}

\author{
Lucila Stange Rezende( ${ }^{(1)}$, Liliam Stange Rezende ${ }^{(2)}$, Sebastião Cezar Radominski( ${ }^{(3)}$ \\ Eduardo dos Santos Paiva ${ }^{(4)}$
}

A fibromialgia (FM) é uma síndrome reumatológica não-inflamatória e não-auto-imune caracterizada por dores musculoesqueléticas generalizadas crônicas, freqüentemente acompanhadas por alteração do sono (sono não restaurador) e por fadiga.

As alterações tireoideanas, especialmente o hipotireoidismo, podem apresentar como características clínicas sinais e sintomas inespecíficos como cansaço, fraqueza, pele seca, dificuldade em concentrar-se e déficit de memória, parestesias, ganho de peso, intolerância ao frio, dores vagas, constipação, dentre outros, que se assemelham a algumas das manifestações da FM, com exceção à presença dos tender points, característicos da última.

Analisando-se a alta prevalência da FM na população geral $(2 \%)$ e a freqüência do seu diagnóstico em clínicas reumatológicas ${ }^{(1,2)}$ e sabendo-se que o hipotireoidismo é também uma condição freqüente ( 1 a $10 \%$ na população geral $)^{(3)}$ que pode simular a FM, torna-se importante avaliar o papel da dosagem do hormônio estimulante da tireóide (TSH) em pacientes com diagnóstico de FM, como um exame de screening.

Durante o período de fevereiro a julho de 2004, foram analisados 108 prontuários de pacientes em acompanhamento no Ambulatório de Fibromialgia do Hospital de Clínicas da Universidade Federal do Paraná (UFPR).

Foram avaliados os prontuários de pacientes que apresentavam diagnóstico de FM, feito por reumatologista, segundo os critérios de classificação do American College of Rheumatology (ACR) de $1990^{(4)}$, e dosagem sérica do TSH, realizada no Laboratório de Medicina Nuclear do Hospital de Clínicas da UFPR.

Os níveis séricos de TSH considerados normais foram de 0,4 a $4,5 \mu \mathrm{UI} / \mathrm{ml}$. Pacientes com níveis séricos de TSH acima de $4,5 \mu \mathrm{UI} / \mathrm{ml}$ foram considerados como tendo hipotireoidismo e pacientes com níveis séricos de TSH abaixo de $0,4 \mu \mathrm{UI} / \mathrm{ml}$, hipertireoidismo.

Outros parâmetros analisados nos pacientes do estudo foram: sexo, idade, queixa de fadiga e/ou alteração do sono, análise do Fibromyalgia Impact Questionnaire (FIQ).

Cento e oito prontuários foram analisados. Cem pacientes eram do sexo feminino e oito do sexo masculino $(92,6 \% \mathrm{e}$ $7,4 \%$, respectivamente). Três subgrupos foram formados com relação ao nível sérico do TSH. Grupo I: FM com TSH normal (75,9\%); Grupo II: FM com TSH elevado (10,2\%); Grupo III: FM com TSH reduzido (13,9\%).

As características da população do estudo são mostradas na Tabela 1 . A faixa etária dos pacientes foi semelhante ( $\mathrm{p}$ $=0,75)$, sendo a idade média de 48,5 anos $( \pm 10,4)$. Não houve diferença estatisticamente significativa entre os valores obtidos no FIQ $(\mathrm{p}=0,929)$. Através do teste de Pearson,

TABELA 1

Características da POPUlação do Estudo

\begin{tabular}{|c|c|c|c|c|c|}
\hline & $\begin{array}{l}\text { Amostra } \\
\text { completa } \\
(n=108)\end{array}$ & $\begin{array}{l}\text { Grupo I } \\
(n=82)\end{array}$ & $\begin{array}{l}\text { Grupo II } \\
(n=11)\end{array}$ & $\begin{array}{l}\text { Grupo III } \\
(n=15)\end{array}$ & ANOVA \\
\hline $\begin{array}{l}\text { Idade } \\
\text { (anos) }\end{array}$ & $\begin{array}{c}48,5 \pm \\
10,4\end{array}$ & $\begin{array}{c}48,04 \pm \\
10,6\end{array}$ & $\begin{array}{c}51,2 \pm \\
12,3\end{array}$ & $\begin{array}{c}49,3 \pm \\
8,02\end{array}$ & $p=0,75$ \\
\hline $\begin{array}{l}\mathrm{TSH}^{*} \text { médio } \\
(\mu \mathrm{UI} / \mathrm{ml})\end{array}$ & $\begin{array}{c}2,46 \pm \\
2,93\end{array}$ & $1,99 \pm 0,95$ & $\begin{array}{c}17,28 \pm \\
27,9\end{array}$ & $\begin{array}{c}0,25 \pm \\
0,18\end{array}$ & \\
\hline $\mathrm{FIQ}^{* *}$ & $\begin{array}{c}51,78 \pm \\
15,69\end{array}$ & $\begin{array}{c}51,58 \pm \\
15,69\end{array}$ & $\begin{array}{c}54,56 \pm \\
18,68\end{array}$ & $\begin{array}{c}50,82 \pm \\
14,57\end{array}$ & $p=0,929$ \\
\hline
\end{tabular}

*TSH: Hormônio estimulante da tireóide

**FIQ: Fibromyalgia Impact Questionnaire

Departamento de Reumatologia do Hospital de Clínicas da Universidade Federal do Paraná (UFPR), Curitiba (PR). Recebido em 13/03/05. Aprovado, após revisão, em 03/11/05.

1. Especializanda em Reumatologia do HC-UFPR - 2. Doutoranda de Medicina do HC-UFPR - 3. Chefe da Residência de Reumatologia do HC-UFPR.

4. Chefe da Especialização de Reumatologia do HC-UFPR, Chefe do Ambulatório de Fibromialgia do HC-UFPR.

Endereço para correspondência: Eduardo dos Santos Paiva, Rua Padre Camargo, 549, conjunto 23/24, Alto da Glória, Curitiba, CEP 80060-240, PR, Brasil, telefone: (41) 233-3598, e-mail:eduevicky@terra.com.br 
não foi observada correlação entre TSH e FIQ. Setenta e seis pacientes apresentaram queixa de fadiga, perfazendo $70,4 \%$ da amostra do estudo. Não foi encontrada relação entre a presença de fadiga e os níveis de TSH (Tabela 2).

A presença de alterações do sono (sono não restaurador)

TABELA 2

Relação Entre Fadiga E Diferentes níveis SÉricos DE TSH*

\begin{tabular}{lc|c|c}
\hline & $\begin{array}{c}\text { Grupo I } \\
(\mathrm{n}=82)\end{array}$ & $\begin{array}{c}\text { Grupo II } \\
(\mathrm{n}=11)\end{array}$ & $\begin{array}{c}\text { Grupo III } \\
(\mathrm{n}=15)\end{array}$ \\
\hline Fadiga Presente & 58 & 8 & 10 \\
Fadiga Ausente & 24 & 3 & 5 \\
\hline
\end{tabular}

*TSH: Hormônio estimulante da tireóideTeste de Fisher:

Grupo I versus Grupo II $p=1,0 \bullet$ Grupo I versus Grupo III $p=0,76 \bullet$ Grupo II

versus Grupo III $p=1,0$

foi relatada por 87 pacientes $(80,5 \%)$. Não foi comprovada relação entre sono não restaurador e os diferentes níveis séricos de TSH nesta amostra.

O diagnóstico de FM é de extrema importância para o médico reumatologista, visto a sua prevalência na população geral. Os diagnósticos diferenciais devem ser rigorosamente avaliados, para se evitar a excessiva valorização do diagnóstico de $\mathrm{FM}^{(5)}$.

Sendo o diagnóstico de FM clínico, questiona-se quais seriam os exames a serem solicitados para estes pacientes, rotineiramente, além daqueles julgados importantes para cada caso. O diagnóstico diferencial de dor crônica generalizada é extenso, incluindo o lúpus eritematoso sistêmico, a osteoartrite poliarticular, a artrite reumatóide, a polimialgia reumática, a síndrome de hipermobilidade, osteomalacia; doenças não-reumatológicas como neoplasias, doenças neurológicas, infecções crônicas e distúrbios psiquiátricos ${ }^{(6)}$. Os resultados obtidos por esse estudo demonstram a importância da dosagem do TSH na avaliação diagnóstica inicial da FM, tendo visto a significativa prevalência de alteração do nível sérico desse hormônio nos pacientes da amostra $(24,1 \%)$.

O significado deste achado permanece incerto, e a relação

\section{REFERÊNCIAS}

1. Goldenberg DL, Burckhardt C, Crofford L: Management of Fibromyalgia Syndrome. JAMA 292: 2388-2395, 2004.

2. Marder WD, Meenan RF, Felsonn DT, et al: The present and future adequacy of Rheumatology manpower: a study of health care needs and physician supply [editorial]. Arthritis Rheum 34: 1209-1217, 1991.

3. Cooper DS: Subclinical hypothyroidism. New Engl J Med 345: 260-265, 2001.

4. Wolfe F, Smythe HA, Yunus MB, et al: The American College of Rheumatology 1990 criteria for the classification of fibromyalgia: report of Multicenter Criteria Committee. Arthritis Rheum 33: 160-172, 1990. entre a tireoidopatia e a FM não apresenta uma definição clara na literatura. Tornou-se comum a investigação de doenças subclínicas da tireóide, especialmente hipotireoidismo, nos pacientes com FM, por se considerar que as duas condições possuem sintomas que podem ser confundidos, como a fadiga, a alteração do sono e as dores difusas.Porém esta relação foi pouco investigada até agora na literatura. Dois estudos referenciados freqüentemente ${ }^{(7,8)}$ foram realizados antes do estabelecimento dos critérios do ACR para o diagnóstico da FM. Em um deles ${ }^{(8)}$, foi pesquisada a prevalência de FM em pacientes com hipotireoidismo primário, e somente cinco pacientes de um total de 100 apresentaram mais de sete tender points (critério utilizado neste estudo). Conclui-se que a freqüência de FM nestes pacientes seria baixa. Estudo(7), retrospectivo, de somente oito pacientes com concomitância dos dois problemas, relatou resolução do quadro álgico após o tratamento com tiroxina.

Não há estudos da relação do hipertireoidismo com FM na literatura.

No nosso estudo, constatou-se que o FIQ variou apenas com a gravidade da FM e não com a alteração dos níveis séricos do hormônio tireoideano.

A conduta frente a pacientes com alterações nos exames de screening de tireoidopatia deve incluir a dosagem repetida de TSH e T4 livre, e a obtenção dos anticorpos antitireoideanos. Existe um interessante relato de uma provável relação da presença destes anticorpos com FM, num estudo em que não foi avaliada a função tireoideana, somente a presença de anticorpos ${ }^{(9)}$. Outros fatores como alterações nos níveis de lipídios, gravidez e disfunção ovulatória devem ser considerados quando da decisão de se começar o tratamento para a doença tireoideana subclínica.

Finalmente, de acordo com os dados expostos, a dosagem sérica do TSH tem papel relevante na avaliação inicial do paciente fibromiálgico visando o seu diagnóstico diferencial ou a associação dessas duas doenças.

5. McCain GA: A cost-effective approach to the diagnosis and treatment of fibromyalgia. Rheum Dis Clin North Am 22: 323349, 1996.

6. Reilly PA: The differential diagnosis of generalized pain. Baillieres Best Pract Res Clin Rheumatol 13: 391-401, 1999.

7. Wilke SW, Leslie SR, Makarowski WS: Hypothyroidism with Presenting Symptoms of Fibrositis. J Rheumatol 8: 626-631, 1981.

8. Carette S, Lefrançois L: Fibrositis and primary hypothyroidism. J Rheumatol 15: 1418-1421, 1988.

9. Ribeiro LS, Proietti FA: Interrelations between fibromyalgia, thyroid autoantibodies, and depression. J Rheumatol 31: 2036-2040, 2004. 